\title{
Periodontal Supportive Care: Aesthetic and Functional Restoration with Acrylic Gingival Veneer
}

\author{
Lim Ghee Seong
}

Department of Restorative Dentistry, Faculty of Dentistry, University of Malaya, 50603 Kuala Lumpur, Malaysia.

\begin{abstract}
This case involved periodontal supportive care after non-surgical management of localized, severe chronic periodontitis (possibly with a history of aggressive periodontitis) and periodontal abscess. This included maintaining the current periodontal health, and rehabilitation of patient's oral function and aesthetic concern using simple, economical and reversible direct restorations without further damaging the patient's dentition for example composite resin as splint. Besides that, to address the patient's complaint of unsightly gaps in between the teeth, an acrylic gingival prosthesis with denture tooth incorporated was constructed.
\end{abstract}

Keywords: Periodontal supportive care, aggressive periodontitis, oral function, aesthetic, composite build up, acrylic gingival prosthesis.

\section{Gingival Prostheses Overview}

Gingival recession is a common oral manifestation due to the progression of periodontal disease, periodontal pocket elimination procedures, resective osseous surgeries and it can also present as one of the healing signs after successful non-surgical management of periodontal disease (1).Loss of interdental papilla is due to the bone loss caused by periodontal disease, leading to wide open and unsightly embrasures between the teeth which would appear in the form of black triangles (1), hence the term 'black triangle syndrome'.(6) It has a relatively high occurrence rate $(1,2)$ and it is a matter of great concern for patients who are self-conscious about esthetics. Some of these patients present with high smile lines, elongated crowns due to root exposure and uneven gingival margins (3). Apart from the unsightly gaps between the teeth, the patients would also experience dentine hypersensitivity to cold air or fluids, and this would further impair oral hygiene maintenance around the area rendering plaque removal difficult $(1,3)$. This manifestation could be a vicious cycle which might ultimately result in tooth loss $(3,4)$. Ignorance of this clinical problem could be a form of negligence. Therefore, the patients need to be well informed about this condition and the possible treatment options.

There are two viable options to correct or manage this problem depending on the severity of the case $(1,5)$. The first option, for mild to moderate gingival defects, could be corrected through surgical means (muco-gingival surgery) thus restoring the original tissue contour and giving the patient an amiable smile $(1,2)$. However, in severe gingival recession where the tissue loss is greater, postsurgical esthetic outcome may be less predictable $(1,2,4,6)$. Such severe gingival recession can be rectified with a simpler option, which involves replacing the loss of gingival tissue with a prostheses known as a gingival veneer. It is also known as gingival mask, party gums, gingival slips, gingival veneer prosthesis, artificial gingiva and gingival replacement unit (1). 
A gingival veneer is defined as a prosthesis that is worn by the patient in the frontal zone (labial part) of the dental arch to restore the mucogingival contour, hence esthetics, in areas where there is periodontal tissue deficiency $(5,7)$. It is not a new treatment modality, as it was first introduced in 1955 by Emslie (8) in a post gingivectomy case, where the gingival veneer was worn to mask the unpleasant appearance of gingival recession after the procedure $(5,8)$. Historically, gingival veneers were used to replace lost periodontal tissues when other treatment modalities (e.g. muco-gingival surgery or other regenerative surgery) showed less favorable or unpredictable results (4). Some of the commonly used materials to construct the gingival prostheses are, porcelains, pink composite resin, pink auto cured, heat cured acrylics, silicone based soft materials and thermoplastic acrylics $(4,5)$.

The gingival veneer has several indications or uses as follows:

- To mask exposed crown margins, root surfaces, implant components, unaesthetic inter-dental dark spaces $(1,5,8,9)$.

- To reduce the elongated clinical crown height (1).

- To block out or obturate the empty spaces (black triangles) between teeth which were previously occupied by interdental papillae. This will avoid food impaction in these areas, improve speech and enable better saliva control $(1,5,8,9)$.

- To fill up the gaps or spaces between fixed restorations (crowns / bridges) and soft tissues, which will prevent air leaking through these spaces, thus improving phonetics $(1,8)$.

- To aid in reduction of root hypersensitivity $(5,8$, 9).

- To provide sufficient cheek and upper lip support to those in need (1).

- To mask the unpleasant dark metal margin of old porcelain fused metal crowns. The metal margin could be exposed in patients who suffered from gingival recession (1).

However, the gingival veneer is contraindicated in patients who exhibit poor oral hygiene maintenance, poor manual dexterity, uncontrolled periodontal disease, caries prone / high caries activity, cigarettes smoking, unmotivated patients and patients with known allergy to materials like acrylic or silicone (1, $5,8,9)$.

There are several types of gingival veneers mentioned in the literature $(1-6,10,11)$, it is categorized mainly into removable and fixed,. Removable veneers are further classified according to the fabrication materials, such as flexible (silicone based) and non-flexible (pink acrylic) materials (6).
Before deciding on whether to prescribe a fixed or removable gingival veneer, a clear understanding of clinical requirement and a sound knowledge on the fabrication materials is required. There are advantages for giving a fixed prosthesis such as patient's comfort, self-confidence is enhanced. However the downside of it is, the difficulties to maintain the oral hygiene and the tissue portions on the prostheses cannot be easily adjusted (12). Whereas with a removable prosthesis, a larger volume of soft tissue loss can be restored and oral hygiene maintenance is relatively easy. With the current fabrication materials, soft tissue ideal contour can be shaped and the missing tissue can be replaced conservatively without touching sound tooth structure (12).

\section{Clinical Case Report}

A 44 year-old gentleman presented to the clinic with complaint of his mobile front teeth and felt that the condition was getting worse He also expressed his concern regarding the appearance of his teeth and felt embarrassed during gatherings with his friends. Besides that, he also mentioned that there was pain on chewing while using his front teeth as the teeth splayed outward. He was very worried that he might eventually lose his front teeth, the presence of the huge unsightly gap resultant from the migration of the teeth due to the periodontal disease and he was unwilling to wear a removable prosthesis. Medically, he was fit and healthy. Past dental history recorded that he was a regular dental attendee. He received a few courses of periodontal treatment which involved non-surgical management such as oral hygiene instructions, root surface instrumentation under local anesthesia adopting full mouth disinfection approach which was a non-surgical periodontal therapy which carried out to prevent re-infection of the bacteria reservoirs mainly from the untreated periodontal pockets (13) and antibiotic (amoxicillin 500mg t.d.s. and metronidazole $400 \mathrm{mg}$ t.d.s.) as adjunct for 5 days, followed by review. During the first meeting, he was not wearing any prostheses. He has a rather unremarkable social history, he was a private employee, non-smoker and only drank alcohol occasionally. There is no abnormalies detected extraorally. Intraorally, the gingivae and oral mucosa appears pink and healthy, however there was localized gingival recession on 11 and 21 exposing $1 / 3$ to $1 / 2$ of the root length due to healing. General probing depth between 5-9mm with no bleeding on probing noted (Figure 1). Patient exhibited excellent oral hygiene. The tooth position of 11 has shifted to the central leaving an unsightly gap between 11 and 12 (Figure 2). Tooth mobility grade 1-2 was 
generally noted with tooth 11 and 21 grade 2 , tooth $12,22,31$ and 41 grade 1 . Besides that, occlusal trauma (fremitus) of 11, 21 was noted as well which worsen the tooth mobility problem. Radiographically (long cone periapical radiograph of 11, 21), there was horizontal bone resorption involving $2 / 3$ of the root length, however no periapical radiolucency was noted. Pulp sensibility test was performed on tooth 12, 11, 21 and all showed vital pulp. Thus, the diagnoses was localized Chronic Moderate Advanced Periodontitis (non-inflammatory) and occlusal trauma of tooth 11, 21.

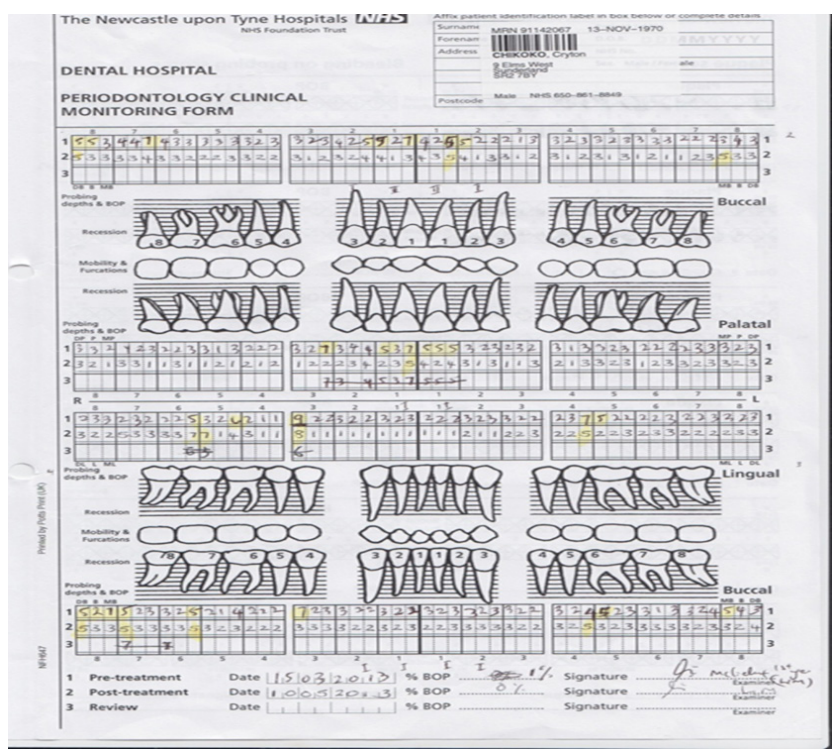

Figure 1: Periodontal Charting Form after 3 Months Post Op Review

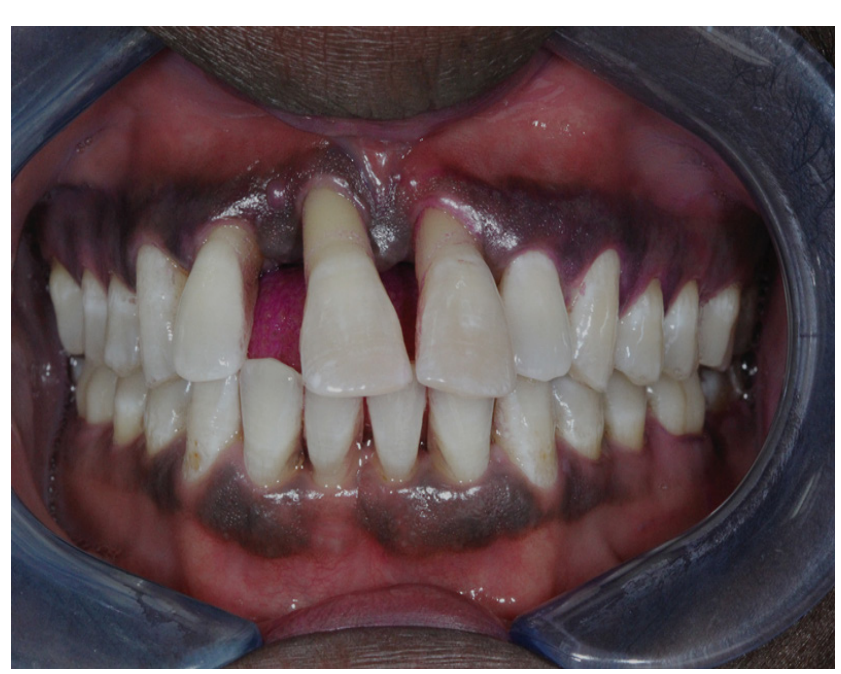

Figure 2: After Periodontal Treatment. Unsightly Gaps in Between the Upper Anterior Teeth.

The objectives of treatment are to address patient's complaints by stabilizing and maintaining the periodontal condition, dentition and provide a stable occlusion while improving the patient's aesthetics. The treatment plan was formulated which includes, close monitoring and maintenance of patient's good oral hygiene; elimination of occlusal trauma involving tooth 11 and 21 by grinding off $1 / 3$ of the enamel of the over erupted 41 and 32 . This is followed by splinting of the lower anterior teeth using orthodontic wire to prevent further eruption of the teeth. Resin composite splint was done on tooth 11 to 21 and composite build ups for tooth 11,21 and 22 to alter the tooth shape and contour for aesthetic purposes (Figure 3). Then, acrylic gingival veneer with a lateral incisor acrylic tooth incorporated (Figure 4). Lastly, recall appointment every 3 months and subsequently every 6 months.

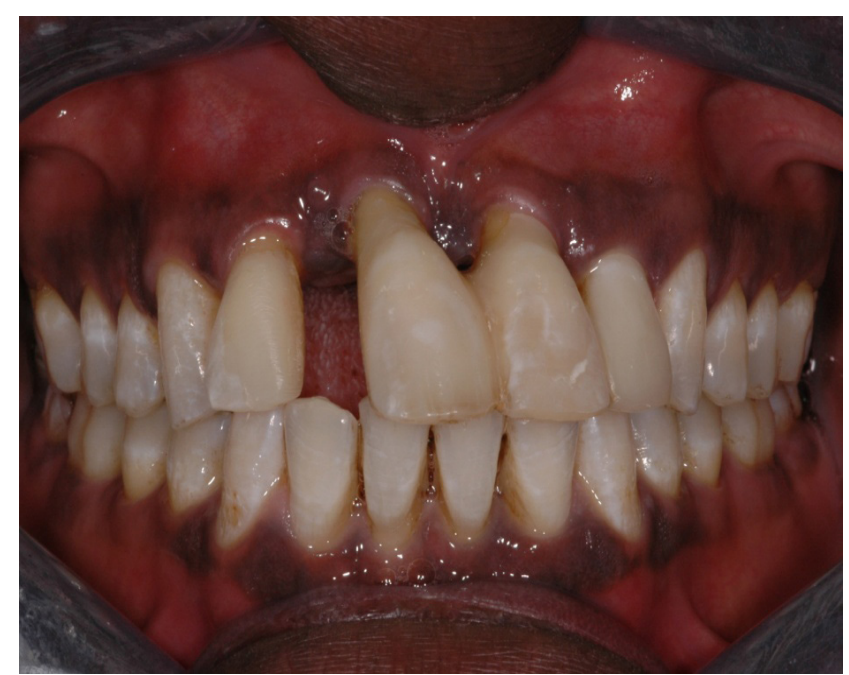

Figure 3: After Resin Composite Build Ups on Tooth 11, 21 and 22 and Splinted (11-21).

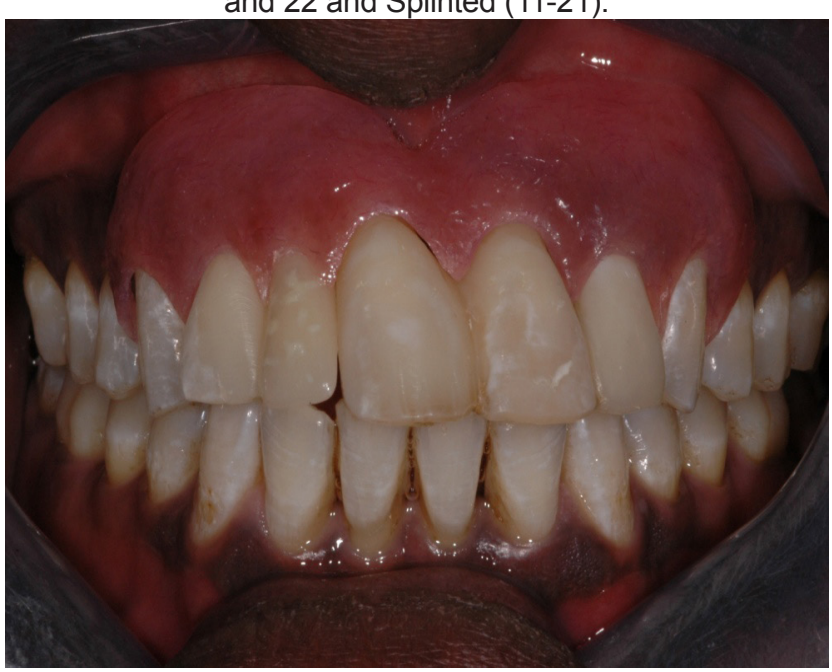

Figure 4: After Insertion of the Upper Gingival Veneer with Tooth 12 Incorporated

\section{Discussion}

The management of the patient's dental problems involved periodontal supportive care which is the 
care given after a lengthy courses of periodontal treatment is completed and during the review visits, oral hygiene was well maintained and there were no sign inflammatory periodontal pockets. In the present case, it involved the care after series of non-surgical management of periodontal disease (Periodontal supportive therapy). The patient's main concerns were the mobility and the pain during mastication using his upper anterior teeth and the unsightly gaps in between the teeth. Therefore, the main aims and objectives of the treatment were to improve function and aesthetics, retaining the upper anterior teeth as long as possible and restoring a stable occlusion. Our treatment plan was determined after lengthy discussion with the patient and the clinician (the author) to ensure that the patient's expectations were met and it was within the clinician's ability to deliver the prescribed treatment to a high standard.

In treated advanced chronic periodontitis, post-treatment generalized recession with loss of interdental papillae is often the tell-tale sign of healing. For this case, normally the mucogingival surgery approach would not give predictable aesthetic results. Therefore a removable gingival veneer is preferred. If the clinician wishes to mask the gingival defect after canine, hard material (non-flexible) like acrylic is not suitable due to the undercuts and would further cause damage to the gingival tissue compared to soft material such as the silicone based material which is more flexible and resilient (12). Furthermore patients would feel more comfortable with flexible materials compared to nonflexible materials (12). However, due to the limitation of the laboratory facilities in the school, acrylic veneer was the only option. The author believed that, with time patient would be able to adapt to the acrylic veneer and with long term review of the patient's oral hygiene and periodontal status, it should not be a problem.

Retention of the prostheses was always questionable. The gingival veneer gains its retention mechanically, with a good utilization of undercuts in between teeth previously occupied by the interdental papillae or between implants just above the gingival line (1). The natural capillary action formed by the saliva (cohesion) also partly contributes to retention of the veneer. The neuromuscular control from the pressure of the lips against the gingival veneer contributes to its retention as well(1).

The author managed to complete the patient's treatment within one year. The author was confident that the ability to achieve functionally satisfying results with good aesthetics was a bonus. The patient is very grateful and happy with the treatment outcome. Based on his feedback during the review visits, the author seem to have managed to meet his expectations. The author has discharged the patient back to his private practitioners for long term monitoring of his periodontal conditions and the prosthesis.

\section{Conclusion}

Gingival veneer can be a useful treatment modality for highly motivated patients with huge gingivae black triangles. It is capable of producing good aesthetic results with lower cost imposed to the patients. However, it is not suitable for all patients, therefore careful case selection is highly recommended before recommending and commencing this treatment on the patients.

\section{Acknowlegement}

I would like to express my gratitude to my Restorative Dentist consultants and supervisors at Newcastle Dental Hospital (United Kingdom) for their dedicated support in supervision of the case and also the supporting staffs including the nurses and prosthodontic lab technicians who were involved in the execution of the treatment.

\section{References}

1. Antony V, Khan R. Gingival Mask-Restoring the Lost Smile. Journal of Dental and Medical Sciences. 2013;5(3):20-2.

2. Patil S, Prabhu V, Danane NR. Gingival Veneer: Mask the Unesthetic. Journal of Indian Society Periodontology. 2011;15(3):284-7.

3. Zalkind M, Hochman N. Alternative Method of Conservative Esthetic Treatment for Gingival Recession. Journal of Prosthetic Dentistry. 1997;77(6):561-3.

4. Barzilay I, Irene T. Gingival Prostheses-A Review.Journal of the Canadian Dental Association. 2003;69(2):74-8.

5. Sinha A, Ravindra S, Bhat S. Gingival Veneer: Non Esthetics to Esthetic Smile. IOSR journal of dental and medical science. 2014; 13(11):269

6. Moldi A, Gala V, Patil V, Desai M, Giri G, Rathod A. Flexible Gingival Veneer: A Quick Cosmetic Solution to Root Coverage-A Case Report. The Internet Journal of Dental Science. 2014;13(1).

7. Ellis S, SharmaP, Harris I. Case Report:Aesthetic Management of a Localised Periodontal Defect with a Gingival Veneer Prosthesis. The European Journal of Prosthodontics and Restorative Dentistry. 2000;8(1):23-6. 
8. Emslie R. A Case of Advanced Periodontitis Complex. Dental Practitioner. 1955;5:432-3.

9. Hickey B, Jauhar S. Gingival veneers. Dental Update. 2009;36(7):422-4, 6, 8.

10. Alani A, Maglad A, Nohl F. The prosthetic management of gingival aesthetics. British dental journal. 2011;210(2):63-9.

11. Choudhari P, Pillai A, Zade R, Amirishetty R, Shetty S. Gingival Veneer: A Novel Technique of Masking Gingival Recession. Journal of Clinical and Diagnostic Research: JCDR. 2015;9(1):ZD12.

12. Vinnakota DN, Akula SR, Kukunoor S, Mempally H. Diverse Modalities of Gingival Replacement: A Report of Three Cases. Contemporary Clinical Dentistry. 2012;3(3):320.
13. Geena K, Esmonde FC, Isao I. A FullMouth Disinfection Approach to Nonsurgical Periodontal Therapy: Prevention of Reinfection from Bacteria Reservoirs. Periodontology 2000, Vol. 36, 2004, 166-178.

\section{Corresponding author:}

\section{Dr. Lim Ghee Seong}

Department of Restorative Dentistry,

Level 5, Dental Specialist and Postgraduate Tower, Faculty of Dentistry, University of Malaya,

50603 Kuala Lumpur

Malaysia.

Tel: +603-79674814

Fax: +603-79674814

E-mail: g.s.lim@um.edu.my 